\title{
Stabilizing Solution for a Discrete-Time Modified Algebraic Riccati Equation in Infinite Dimensions
}

\author{
Viorica Mariela Ungureanu \\ “Constantin Brâncuşi” University of Tirgu-Jiu, B-dul Republicii No. 1, 210152 Târgu Jiu, Romania \\ Correspondence should be addressed to Viorica Mariela Ungureanu; lvungureanu@yahoo.com
}

Received 12 March 2015; Revised 19 May 2015; Accepted 26 May 2015

Academic Editor: Driss Boutat

Copyright (C) 2015 Viorica Mariela Ungureanu. This is an open access article distributed under the Creative Commons Attribution License, which permits unrestricted use, distribution, and reproduction in any medium, provided the original work is properly cited.

\begin{abstract}
We provide necessary and sufficient conditions for the existence of stabilizing solutions for a class of modified algebraic discretetime Riccati equations (MAREs) defined on ordered Banach spaces of sequences of linear and bounded operators. These MAREs arise in the study of linear quadratic (LQ) optimal control problems for infinite-dimensional discrete-time linear systems (DTLSs) affected simultaneously by multiplicative white noise $(\mathrm{MN})$ and Markovian jumps (MJs). Unlike most of the previous works, where the detectability and observability notions are key tools for studying the global solvability of MAREs, in this paper the conditions of existence of mean-square stabilizing solutions are given directly in terms of system parameters. The methods we have used are based on the spectral theory of positive operators and the properties of trace class and compact operators. Our results generalise similar ones obtained for finite-dimensional MAREs associated with stochastic DTLSs without MJs. Also they complete and extend (in the autonomous case) former investigations concerning the existence of certain global solutions (as minimal, maximal, and stabilizing solutions) for generalized discrete-time Riccati type equations defined on infinite-dimensional ordered Banach spaces.
\end{abstract}

\section{Introduction}

In recent years, the study of optimal control problems associated with stochastic systems with Markovian regime switching is of particular interest to researchers due to their various applications in finance, biology, engineering, and so forth. Even in the case of linear systems, the optimization problems become considerably hard, when we are in infinite dimensions and/or the Markovian process has infinite state space (see, e.g., [1-5] and the references therein). As we know from the LQ optimal control theory of DTLSs with MN and MJs (see, e.g., [5-7]), the design of the optimal control is closely related to the existence of a stabilizing solution (SS) for an associated generalized discrete-time Riccati equation (GDTRE). In this paper, we investigate the solution properties for a class of MAREs of control associated with autonomous infinite-dimensional DTLSs with MN and infinite MJs. These MAREs are time-invariant versions of the infinitedimensional GDTREs studied in [8]. For a detailed treatment of finite-dimensional GDTREs, the reader can consult $[6,7]$ and the references therein. The problem of existence of SSs for infinite-dimensional GDTREs associated with stabilizable DTLSs with infinite MJs was investigated in $[3,5,9,10]$ under either stochastic detectability or observability hypotheses. In [8], a set of necessary and sufficient conditions for the existence of SSs is expressed in terms of feasibility of some linear matrix inequalities (LMIs) system.

The general theory of GDTREs applies to our MAREs but new special results are strongly expected in the autonomous case. For example, in finite dimensions it is proved (see [11]) that necessary and sufficient conditions for the existence of stabilizing solutions for MAREs can be given directly in terms of system parameters. They consist in verifying whether the stochastic system is stabilizable and $\lambda=1$ is not an unobservable eigenvalue for a pair of associated operators. Although it is not always possible to give an infinite-dimensional version of a finite-dimensional result (as an example, we recall the researches proving that Hautus Lemma does not work in infinite dimensions $[12,13])$, in this paper we have tried to do this for Theorem 12 from [11].

The approach we propose is based on operator theory tools and the properties of positive operators and their 
adjoints. Using the results from [8], we prove that conditions similar to those in [11] are necessary for the existence of SSs for infinite-dimensional MAREs (see Theorem 10). To obtain sufficient conditions, we extend the class of eigenvalues $\lambda$ that must not be unobservable to the set $[1, \infty)$ and we impose additional constraints for the coefficients of the DTLSs with MN and MJs (see Theorems 11 and 13). When being applied to finite-dimensional DTLSs with MN, our results recover the ones in [11]. However, Corollary 14 seems to be new for finitedimensional DTLSs with finite Markovian jumps. As we have shown in Example 1, it provides necessary and sufficient conditions for the existence of SSs for MAREs associated with DTLSs with MJs which are neither stochastically observable nor stochastically detectable. In this case, the results like those from $[2,5,10]$ do not work, while a LMIs approach would lead to a large number of operations for systems of large dimensions.

The paper is organized as follows. In Section 2, we introduce some basic notation and terminology and we formulate the problem. Section 3 briefly reminds us about certain properties of nuclear (trace class) and compact operators and extends to a more general framework, the well-known result: "the dual space of trace-class operators space is isomorphic to the set of bounded operators." Also, we define the notions of maximal and stabilizing solution for a MARE and we show how to apply the results from [8] to the MAREs discussed in this paper. In Section 4, we obtain the main results. We first introduce the notion of unobservable eigenvalue for a pair of operators, by using spectral properties of positive operators. Then, we provide necessary and sufficient conditions for the existence of stabilizing solutions to MAREs (see Theorems 10, 11, and 13). To obtain the sufficient conditions from Theorems 11 and 13, we assume additional hypotheses like compactness of the coefficients or trace-class membership. Corollary 14 shows that the finite-dimensional results from [11] are direct consequences of Theorems 10, 11, and 13.

Section 5 is devoted to numerical examples. We study three examples, which cover the cases of MAREs associated with finite-dimensional DTLSs with finite MJs and infinitedimensional DTLSs with infinite MJs. As mentioned above, Example 1 shows the advantages of Corollary 14 over previously published results $[2,5,10]$.

Finally, the last section provides conclusions and further research lines.

\section{Notations and Statement of the Problem}

Let $H, U$, and $V$ be real separable Hilbert spaces. We denote by $L(H, U)$ the real Banach space of linear and bounded operators from $H$ into $U$ and by $S(H)$ the Banach subspace of $L(H):=L(H, H)$, formed by all self-adjoint operators. As usual ${ }^{*}$ denotes either the adjoint of a linear and bounded operator or the dual of a Banach space. We will write $\langle\cdot, \cdot\rangle$ for the inner product and $\|\cdot\|$ for norms of elements and operators, unless otherwise is indicated. An operator $A \in$ $L(H)$ is called nonnegative and we write $A \geq 0$, if $A$ is selfadjoint and $\langle A x, x\rangle \geq 0$ for all $x \in H$.

Let $\mathscr{Z}$ be an interval of integers, which may be finite or infinite. If $(B,\|\cdot\|)$ is a real Banach space, then $l_{B}^{\mathscr{E}}=$ $\left\{g=\{g(i) \in B\}_{i \in \mathscr{F}},\|g\|_{\mathscr{X}}=\sup _{i \in \mathscr{E}}\|g(i)\|<\infty\right\}$ is a real linear space with the usual termwise addition and (real) scalar multiplication. Moreover, $l_{B}^{\mathscr{I}}$ is a Banach space when endowed with the norm $\|\cdot\|_{\mathscr{X}}$. If $B$ is $L(U, H)$ or $S(H)$, then $l_{B}^{\mathscr{E}}$ will be denoted by $l_{L(U, H)}^{\mathscr{E}}$ or $l_{S(H)}^{\mathscr{E}}$. Let $\mathbb{N}$ be the set of natural numbers and $\mathbb{N}^{*}=\mathbb{N}-\{0\}$. We denote by $l\left(\mathbb{N}, l_{S(H)}^{\mathscr{I}}\right)$ the Banach space $l_{l_{S(H)}^{\mathscr{I}}}^{\mathscr{I}}$ obtained from $l_{B}^{\mathscr{I}}$ by replacing $\mathscr{Z}$ and $B$ by $\mathbb{N}$ and $l_{S(H)}^{\mathscr{I}}$, respectively.

An element $X \in l_{S(H)}^{\mathscr{E}}$ is said to be nonnegative (we write $X \geq 0)$ iff $X(i) \geq 0$ for all $i \in \mathscr{Z}$. The cone $\mathscr{K}_{H}^{\mathscr{Z}}$ of all nonnegative elements of $l_{S(H)}^{\mathscr{E}}$ introduces the following order on $l_{S(H)}^{\mathscr{Z}}$ :

$$
X \geq Y \quad \text { iff } X-Y \in \mathscr{K}_{H}^{\mathscr{X}} .
$$

Let $I_{H}$ be the identity operator on $H$ and let $\Phi_{H}$ be the element $\left\{\Phi_{H}(i)=I_{H}\right\}_{i \in \mathscr{Z}}$ of $l_{S(H)}^{\mathscr{I}}$. We say that $X \in l_{S(H)}^{\mathscr{Z}}$ is positive and we write $X>>0$, if there is $\gamma>0$ such that $X \geq$ $\gamma \Phi_{H}$. A sequence $\left\{X_{n}\right\}_{n \in \mathbb{N}} \subset l_{S(H)}^{\mathscr{x}}$ is called nonnegative if $X_{n} \in \mathscr{K}_{H}^{\mathscr{E}}$ for all $n \in \mathbb{N}$ and uniformly positive (we write $X_{n} \gg>0$ ) if there is $\gamma>0$ such that $X_{n} \geq \gamma \Phi_{H}$ for all $n \in \mathbb{N}$.

A linear and bounded operator $\Gamma \in L\left(l_{S(H)}^{\mathscr{E}}, l_{S(U)}^{\mathscr{E}}\right)$ is called positive iff $\Gamma\left(\mathscr{K}_{H}^{\mathscr{E}}\right) \subset \mathscr{K}_{U}^{\mathscr{E}}$. For $A \in l_{L(U, H)}^{\mathscr{I}}$ and $B \in l_{L(H, U)}^{\mathscr{E}}$, we define the product $A B \in l_{L(H)}^{\mathscr{I}}$ by $(A B)(i)=A(i) B(i), i \in \mathscr{Z}$.

Now let us introduce two special notations on spaces $l_{B}^{\mathscr{E}}$. If $P \in l_{L(H, U)}^{\mathscr{I}}$, then $P^{[*]}$ denotes the element of $l_{L(U, H)}^{\mathscr{I}}$ defined by $P^{[*]}(i)=P(i)^{*}, i \in \mathscr{Z}$ (i.e., the $i$ th component of $P^{[*]}$ is the adjoint operator of $P(i))$. Also, if $P \in l_{S(H)}^{\mathscr{E}}$ is such that $P(i)^{-1}$ exists for all $i \in \mathscr{Z}$, then $P^{[-1]}=\left\{P(i)^{-1}, i \in \mathscr{Z}\right\}$. Note that $P^{[-1]}$ may not belong to $l_{S(H)}^{\mathscr{E}}$.

Let $(\Omega, \mathscr{F}, P)$ be a probability space. For any integrable random variable $\xi$ on $(\Omega, \mathscr{F}, P)$, we write $E[\xi]$ for its expectation (mean). We will denote by $L^{2}(\Omega, H)$ the Hilbert space of all $H$ valued random variables $\xi$ with the property $E\left(\|\xi\|^{2}\right)<$ $\infty$. If $\xi$ and $\eta$ are integrable real valued random variables, we denote by $E[\xi \mid \eta=x]$ the conditional expectation on the event $\eta=x$. We recall that, by definition,

$$
E[\xi \mid \eta=x]=\frac{1}{P\{\eta(\omega)=x\}} \int \chi_{\{\eta(\omega)=x\}} \xi(\omega) P(d \omega),
$$

if $P\{\omega / \eta(\omega)=x\}>0$, and $E[\xi \mid \eta=x]=0$ otherwise.

Let $\left\{\eta_{t}\right\}_{t \in \mathbb{N}}$ be a homogeneous Markov chain on $(\Omega, \mathscr{F}, P)$ with the state space $\mathscr{E}$, the transition probability matrix

$$
P=\left\{p_{i, j}:=P\left(\eta_{t+1}=\left.j\right|_{\eta_{t}=i}\right)\right\}_{(i, j) \in \mathscr{E} \times \mathscr{E}}, \quad t \in \mathbb{N},
$$

and the property that $P\left(\eta_{t}=i\right)>0, t \in \mathbb{N}, i \in \mathscr{Z}$. The last condition ensures the nontrivial computation of the conditional mean $E\left[\xi \mid \eta_{t}=i\right], \xi \in L^{2}(\Omega, H)$. 
Throughout this paper, we assume that the following hypotheses hold.

(i) $r \in \mathbb{N}^{*}$ is fixed, $A_{p} \in l_{L(H)}^{\mathscr{I}}, B_{p} \in l_{L(U, H)}^{\mathscr{I}}, p=$ $0, \ldots, r$, and $M \in l_{S(H)}^{\mathscr{I}}, L \in l_{L(U, H)}^{\mathscr{I}}$, and $R \in l_{S(U)}^{\mathscr{I}}$ are such that

$$
\begin{aligned}
& R>0, \\
& Q=\left(\begin{array}{cc}
M & L \\
L^{[*]} & R
\end{array}\right) \geq 0 .
\end{aligned}
$$

(ii) $\left\{w_{p}(t)\right\}_{t \in \mathbb{N}}, p=1, \ldots, r$, are sequences of real valued, zero mean, square integrable random variables on $(\Omega, \mathscr{F}, P)$ having the properties that $w(t)=\left(w_{1}(t), w_{2}(t), \ldots, w_{r}(t)\right)^{T}, t \in \mathbb{N}$, is a sequence of independent random vectors, $E\left[w(t) w(t)^{T}\right]=I_{r}, n \in \mathbb{N}$ and $\left\{\eta_{t}\right\}_{t \in \mathbb{N}}$, and $\{w(t)\}_{t \in \mathbb{N}}$ are independent. (Here ${ }^{T}$ denotes the transposition.)

Let $\mathscr{F}_{t}$ and $\mathscr{G}_{t}$ be the $\sigma$-algebras generated by $\left\{w_{1}(k)\right.$, $\left.w_{2}(k), \ldots, w_{r}(k), 0 \leq k \leq t\right\}$ and $\left\{\eta_{k}, 0 \leq k \leq t\right\}$. We introduce the $\sigma$-algebra $\mathscr{H}_{t}, t \in \mathbb{N}$ defined by $\mathscr{H}_{0}=\mathscr{G}_{0}$ and $\mathscr{H}_{t}=\mathscr{F}_{t-1} \vee \mathscr{G}_{t}$ if $t \geq 1$.

We consider the stochastic system with MNs and MJs

$$
\begin{aligned}
x(t+1)= & {\left[A_{0}\left(\eta_{t}\right)+\sum_{p=1}^{r} w_{p}(t) A_{p}\left(\eta_{t}\right)\right] x(t) } \\
& +\left[B_{0}\left(\eta_{t}\right)+\sum_{p=1}^{r} w_{p}(t) B_{p}\left(\eta_{t}\right)\right] u(t), \\
& t \geq t_{0}, t \in \mathbb{N} \\
x\left(t_{0}\right)= & x \in H
\end{aligned}
$$

denoted $(A, B)$ and the cost functional

$$
\begin{aligned}
& J\left(u ; t_{0}, x, i\right)=\sum_{t=t_{0}}^{\infty} E\left[\left(\left\langle M\left(\eta_{t}\right) x(t), x(t)\right\rangle\right.\right. \\
& \left.\quad+2\left\langle L\left(\eta_{t}\right) u(t), x(t)\right\rangle+\left\langle R\left(\eta_{t}\right) u(t), u(t)\right\rangle\right) \mid \eta_{t} \\
& \quad=i] .
\end{aligned}
$$

An optimal control problem (O) associated with (5) and (7) consists in minimizing the cost functional $J\left(u ; t_{0}, x, i\right), i \in$ $\mathscr{Z}$, subject to (5), over the set $\mathscr{U}_{t_{0}, x}$ of admissible controls $u=$ $\{u(t)\}_{t \geq t_{0}, n \in \mathbb{N}}$ with the property that $u(t), t \geq t_{0}$, are $U$-valued, $\mathscr{H}_{t}$-measurable random variables such that

$$
\sup _{t \geq t_{0}} E\left[\left.\|u(t)\|^{2}\right|_{\eta_{t_{0}}=i}\right]<\infty
$$

and $J\left(u ; t_{0}, x, i\right)<\infty$ for all $i \in \mathscr{Z}$.

$$
\begin{aligned}
& \text { Let } \mathfrak{F}: l_{S(H)}^{\mathscr{Z}} \rightarrow l_{S(H)}^{\mathscr{Z}} \text {, and } \\
& \qquad \mathfrak{F}(X)(i)=\sum_{j \in \mathscr{X}} p_{i j} X(j), \quad i \in \mathscr{Z} .
\end{aligned}
$$

As we know from $[5,7],(\mathcal{O})$ has a solution $u_{\text {opt }}$ if the following modified algebraic Riccati equation (MARE):

$$
\begin{aligned}
X(i)= & \sum_{p=0}^{r} A_{p}^{*}(i) \mathfrak{F}(X)(i) A_{p}(i)+M(i) \\
& -\left[L(i)+\sum_{p=0}^{r} A_{p}^{*}(i) \mathfrak{E}(X)(i) B_{p}(i)\right] \\
& \cdot\left[R(i)+\sum_{p=0}^{r} B_{p}^{*}(i) \mathfrak{E}(X)(i) B_{p}(i)\right]^{-1} \\
& \cdot\left[L^{*}(i)+\sum_{p=0}^{r} B_{p}^{*}(i) \mathfrak{E}(X)(i) A_{p}(i)\right],
\end{aligned}
$$

has a mean-square stabilizing solution (see Definition 8).

Our problem is to provide a set of necessary and sufficient conditions for the existence of a stabilizing solution for MARE (10).

\section{Preliminary Results}

3.1. Sequences of Nuclear and Compact Operators. As in [9], we denote by $S_{1}(H)$ the Banach space of all nuclear operators from $S(H)$. It is known that $S_{1}(H)$ is a Banach space when endowed with the nuclear norm $\|T\|_{1}=\operatorname{Tr}\left[\sqrt{T^{*} T}\right]$. Here, $\operatorname{Tr}[\cdot]$ is the trace operator. It is well know (see, e.g., $[14,15]$ ) that

$$
\|\cdot\| \leq\|\cdot\|_{1} \cdot
$$

For further properties of nuclear operators, the reader is referred to $[9,14-16]$. It is known that the linear subspace $\mathcal{N}_{H}=\left\{T \in l_{S(H)}^{\mathscr{Z}},\||T|\|_{1}=\sum_{i \in \mathscr{Z}}\|T(i)\|_{1}<\infty\right\}$ of $l_{S(H)}^{\mathscr{Z}}$ is a Banach space (see [9]) when endowed with the norm $\||\cdot|\|_{1}$. An easy computation (see [9]) shows that

$$
\|\cdot\|_{\mathscr{D}} \leq\||\cdot|\|_{1} \cdot
$$

In the sequel we sometimes use a special element of $\mathcal{N}_{H}$, defined for any $i \in \mathscr{Z}$ and $x \in H$ by

$$
\begin{aligned}
& { }_{i, x} \widehat{P}(j)=0 \quad \text { if } j \neq i, \\
& { }_{i, x} \widehat{P}(i)=x \otimes x
\end{aligned}
$$

Here, $x \otimes x \in S_{1}(H)$ is defined by $x \otimes x(u)=\langle u, x\rangle x$.

Let $\mathscr{C}_{H}$ denote the set all compact operators from $S(H)$. Then, $\mathscr{C}_{H}^{\mathscr{I}}$ will be the subset of $l_{S(H)}^{\mathscr{I}}$ formed by sequences of compact operators; that is, $\mathscr{C}_{H}^{\mathscr{Z}}=\left\{X \in l_{S(H)}^{\mathscr{I}} \mid X(i) \in \mathscr{C}_{H}\right.$ for all $i \in \mathscr{Z}$ \}. 
It is well known that $S_{1}(H)$ is dense in $\mathscr{C}_{H}$ in the uniform operator topology (see, e.g., Problem 5.69 from [15]). The following lemma, whose proof is a simple exercise for the reader, states that a similar result remains true for finite sequences of nuclear and compact operators.

Lemma 1. If $\mathscr{Z}$ is finite, then $\mathcal{N}_{H}$ is dense in $\mathscr{C}_{H}^{\mathscr{Z}}$ with respect to $\|\cdot\|_{\mathscr{X}}$.

Remark 2. In the case when $\mathscr{Z}$ is infinite, the above result is not true as it is proved by the following counter example. Let $H=\mathbb{R}$ and let $\mathscr{Z}=\mathbb{N}$. Then, $\mathscr{C}_{H}^{\mathscr{Z}}=l_{S(\mathbb{R})}^{\mathscr{E}}=l_{\infty}(\mathbb{Z})$ and $\mathscr{N}_{H}=$ $l_{1}(\mathbb{Z})$. Since $l_{1}(\mathbb{Z})$ is separable and $l_{\infty}(\mathbb{Z})$ is not, it follows that $l_{1}(\mathbb{Z})$ is not dense in $l_{\infty}(\mathbb{Z})$. Thus, $\mathcal{N}_{H}$ is not dense in $\mathscr{C}_{H}^{\mathscr{Z}}$.

Proposition 3. The dual space $\left(\mathcal{N}_{H}\right)^{*}$ of the Banach space $\mathcal{N}_{H}$ is isometrically isomorphic with $l_{S(H)}^{\mathscr{E}}$. The isomorphism $\psi$ : $l_{S(H)}^{\mathscr{I}} \rightarrow\left(\mathcal{N}_{H}\right)^{*}$ is defined by $\psi(Y)=\phi_{Y}$, where $\phi_{Y}$ is the linear functional:

$$
\phi_{Y}(T)=\sum_{i \in Z} \operatorname{Tr}[T(i) Y(i)], \quad T \in \mathcal{N}_{H} .
$$

Proof. Let $Y \in l_{S(H)}^{\mathscr{I}}$ and $T \in \mathcal{N}_{H}$ be arbitrarily chosen. The following inequalities:

$$
\begin{aligned}
\left|\phi_{Y}(T)\right| & \leq \sum_{i \in \mathscr{E}}|\operatorname{Tr}[T(i) Y(i)]| \leq \sum_{i \in \mathscr{E}}\|T(i) Y(i)\|_{1} \\
& \leq \sum_{i \in \mathscr{E}}\|Y(i)\|\|T(i)\|_{1} \leq\|Y\|_{\mathscr{E}} \sum_{i \in \mathscr{E}}\|T(i)\|_{1} \\
& \leq\|Y\|_{\mathscr{E}}\|T \mid\|_{1},
\end{aligned}
$$

show that $\phi_{Y} \in\left(\mathcal{N}_{H}\right)^{*}$ and

$$
\left\|\phi_{Y}\right\| \leq\|Y\|_{\mathscr{E}} .
$$

We will prove that $Y \rightarrow \phi_{Y}$ is the isometric isomorphism that maps $l_{S(H)}^{\mathscr{Z}}$ into $\left(\mathcal{N}_{H}\right)^{*}$. Let $\phi \in\left(\mathcal{N}_{H}\right)^{*}$. For any $i \in \mathscr{Z}$, we define $Y(i) \in S(H)$ by

$$
\langle Y(i) x, x\rangle=\phi\left({ }_{i, x} \widehat{P}\right), \quad x \in H .
$$

Obviously, $|\langle Y(i) x, x\rangle| \leq\|\phi\|\left\|\left.\right|_{i, x} \widehat{P} \mid\right\|_{1}=\|\phi\|\|x\|^{2}$. Hence $\|Y(i)\| \leq\|\phi\|, i \in \mathscr{Z}$. It follows that $Y=\{Y(i)\}_{i \in \mathscr{E}} \in l_{S(H)}^{\mathscr{F}}$ and

$$
\|Y\|_{\mathscr{L}} \leq\|\phi\| \text {. }
$$

On the other hand, a simple computation shows that

$$
\langle Y(i) x, x\rangle=\sum_{j \in \mathscr{T}} \operatorname{Tr}\left[\left(_{i, x} \widehat{P}\right)(j) Y(j)\right]
$$

and (17) and (14) imply that

$$
\phi\left({ }_{i, x} \widehat{P}\right)=\phi_{Y}\left({ }_{i, x} \widehat{P}\right)
$$

for all $x \in H, i \in \mathscr{Z}$. It is well known that every $X \in S_{1}(H)$ can be written as a sum $\sum_{n \in \mathbb{N}} \lambda_{n} x_{n} \otimes x_{n}$, where the series is $\|\cdot\|_{1}$-convergent and $x_{n} \in H, n \in \mathbb{N}[15]$. Hence, the element $\widetilde{X}_{i} \in l_{S(H)}^{\mathscr{E}}$, defined by $\widetilde{X}_{i}(i)=X$ and $\widetilde{X}_{i}(j)=0$, for $j \neq i$, $j \in \mathscr{Z}$, can be written as $\sum_{n \in \mathbb{N}} \lambda_{n}\left({ }_{i}, x_{n} \widehat{P}\right)$ where the series is $\||\cdot|\|_{1}$-convergent. Then, (20) and the $\||\cdot|\|_{1}$-continuity of $\phi$ and $\phi_{Y}$ ensure that

$$
\phi\left(\widetilde{X}_{i}\right)=\phi_{Y}\left(\widetilde{X}_{i}\right)
$$

for all $X \in S_{1}(H)$. Now, for an arbitrary $\mathbf{X} \in \mathcal{N}_{H}$, we define the sequence $X_{n} \in \mathcal{N}_{H}, n \in \mathbb{N}, X_{n}(i):=\mathbf{X}(i)$, if $|i| \leq n$, $i \in \mathscr{Z}$ and $X_{n}(i):=0$ for $|i| \geq n, i \in \mathscr{Z}$. It is not difficult to see that $X_{n}, n \in \mathbb{N}$ converges to $\mathbf{X}$ in $\||\cdot|\|_{1}$. From (21), it follows easily that $\phi\left(X_{n}\right)=\phi_{Y}\left(X_{n}\right)$ for all $n \in \mathbb{N}$. Using again the $\||\cdot|\|_{1}$-continuity of $\phi$ and $\phi_{Y}$, we get $\phi(\mathbf{X})=\phi_{Y}(\mathbf{X})$. Therefore, $\phi=\phi_{Y}$ and combining (16) and (18), it follows that $\left\|\phi_{Y}\right\|=\|Y\|_{\mathscr{E}}$. We conclude that the map $\psi$ is an isometric isomorphism from $\left(\mathcal{N}_{H}\right)^{*}$ to $l_{S(H)}^{\mathscr{E}}$. The proof is complete.

Remark 4. A direct consequence of (17) and the above lemma is the following property of the isomorphism $\psi$.

$\phi \in\left(\mathcal{N}_{H}\right)^{*}$ is a positive functional (i.e., $\phi(X) \geq 0$ for any $\left.X \in \mathcal{N}_{H}, X \geq 0\right)$, if and only if $\psi^{-1}(\phi):=Y \geq 0$.

3.2. Maximal and Stabilizing Solutions of MARE. In this section, we recall some definitions and results which we frequently use in the rest of the paper. To be consistent with previous publications (see, e.g., $[7,8]$ and the references therein) concerning discrete-time Riccati equations associated with DTLSs with MN and MJs, we introduce the following notation. For all $X \in l_{S(H)}^{\mathscr{E}}$, let

$$
\begin{aligned}
\Pi_{1}(X)(i) & :=\sum_{p=0}^{r} A_{p}^{*}(i) \mathfrak{E}(X)(i) A_{p}(i), \\
\Pi_{2}(X)(i) & :=\sum_{p=0}^{r} A_{p}^{*}(i) \mathfrak{E}(X)(i) B_{p}(i), \\
\Pi_{3}(X)(i) & :=\sum_{p=0}^{r} B_{p}^{*}(i) \mathfrak{E}(X)(i) B_{p}(i), \\
\Pi(X) & =\left(\begin{array}{cc}
\Pi_{1}(X) & \Pi_{2}(X) \\
\Pi_{2}(X)^{[*]} & \Pi_{3}(X)
\end{array}\right) .
\end{aligned}
$$

We see that $\Pi_{1} \in L\left(l_{S(H)}^{\mathscr{E}}\right), \Pi_{2} \in L\left(l_{S(H)}^{\mathscr{E}}, l_{L(U, H)}^{\mathscr{I}}\right), \Pi_{3} \in L\left(l_{S(H)}^{\mathscr{E}}\right.$, $\left.l_{S(U)}^{\mathscr{I}}\right)$, and $\Pi \in L\left(l_{S(H)}^{\mathscr{E}}, l_{S(H \times U)}^{\mathscr{I}}\right)$ is a positive operator. Then, MARE (10) can be equivalently rewritten as

$$
X=\mathscr{R}(X),
$$

where, for all $X \in \operatorname{Dom} \mathscr{R}=\left\{X \in l_{S(H)}^{\mathscr{D}} \mid\left(R+\Pi_{3} X\right)(i)\right.$ is invertible for all $i \in \mathscr{Z}\}$,

$$
\begin{aligned}
\mathscr{R} & (X) \\
= & \Pi_{1}(X)+M \\
& -\left(L+\Pi_{2}(X)\right)\left(R+\Pi_{3}(X)\right)^{[-1]}\left(L+\Pi_{2}(X)\right)^{[*]} .
\end{aligned}
$$


Following [8], we define the dissipation operator

$$
\begin{aligned}
& D^{\Sigma}: l\left(\mathbb{N}, l_{S(H)}^{\mathscr{Z}}\right) \longrightarrow l\left(\mathbb{N}, l_{S(H)}^{\mathscr{I}}\right), \\
& D^{\Sigma}(X)(t) \\
& \quad=\left(\begin{array}{cc}
\Pi_{1}(X(t+1))+M-X(t) & L+\Pi_{2}(X(t+1)) \\
\left(L+\Pi_{2}(X(t+1))\right)^{[*]} & R+\Pi_{3}(X(t+1))
\end{array}\right)
\end{aligned}
$$

and the subsets $\Gamma^{\Sigma}$ and $\widetilde{\Gamma}^{\Sigma}$ of $l\left(\mathbb{N}, l_{S(H)}^{\mathscr{Z}}\right)$

$$
\begin{aligned}
\Gamma^{\Sigma} & =\left\{X \in l\left(\mathbb{N}, l_{S(H)}^{\mathscr{Z}}\right) \mid R+\Pi_{3}(X(t+1))\right. \\
& \left.>>0, D^{\Sigma}(X)(t) \geq 0, t \in \mathbb{N}\right\} \\
\widetilde{\Gamma}^{\Sigma} & =\left\{X \in l\left(\mathbb{N}, l_{S(H)}^{\mathscr{Z}}\right) \mid D^{\Sigma}(X)(t)>>0\right\} .
\end{aligned}
$$

Lemma 28 from [8] (which is an operatorial version of the Schur complement Lemma) ensures that $X \in \widetilde{\Gamma}^{\Sigma}$ iff (if and only if) $\mathscr{R}(X)-X>>0$.

Definition 5. (a) We say that $X \in l_{S(H)}^{\mathscr{I}}$ is a solution of MARE (10) if $\left(R+\Pi_{3} X\right)(i)$ is invertible for all $i \in \mathscr{Z}$ and $X$ satisfies (10).

(b) We say that a solution $X$ of MARE (10) is maximal if $X \geq \widehat{X}(t), t \in \mathbb{N}$, for arbitrary $\{\widehat{X}(t)\}_{t \in \mathbb{N}} \in \Gamma^{\Sigma}$.

Now, for any $W \in l_{L(H, U)}^{\mathscr{Z}}$, we define

$$
\begin{gathered}
\Pi_{W}(X)=\sum_{p=0}^{r}\left(A_{p}(i)+\left(B_{p} W\right)(i)\right)^{*} \mathfrak{E}(X)(i) \\
\cdot\left(A_{p}(i)+B_{p} W(i)\right), \\
Q_{W}=M+W^{[*]} L^{[*]}+L W+W^{[*]} R W .
\end{gathered}
$$

Obviously, $\Pi_{W} \in L\left(l_{S(H)}^{\mathscr{Z}}\right)$ is a positive operator.

As in [8], let $T_{W}\left(t, t_{0}\right)=\left(\Pi_{W}\right)^{t-t_{0}}, t \geq t_{0}, t, t_{0} \in \mathbb{N}$, be the evolution operator defined by $\Pi_{W}$. We say that $T_{W}\left(t, t_{0}\right)$, is exponentially stable iff there are $\beta \geq 1, \alpha \in(0,1)$ such that

$$
\left\|T_{W}\left(t, t_{0}\right)\right\| \leq \beta \alpha^{t-t_{0}}
$$

for all $t \geq t_{0}, t, t_{0} \in \mathbb{N}$ or, equivalently, iff $\rho_{\Pi_{W}}<1$, where $\rho_{\Pi_{W}}$ denotes the spectral radius of $\Pi_{W}$.

In view of Theorem 14 from [8], we have the following.

Definition 6. The linear and positive operator $\Pi$ is stabilizable if there is $W \in l_{L(H, U)}^{\mathscr{Z}}$ such that the evolution operator $T_{W}\left(t, t_{0}\right), t \geq t_{0}, t, t_{0} \in \mathbb{N}$, is exponentially stable.

Since (4) and (22) imply that $0 \in \Gamma^{\Sigma}$, the following proposition is a direct consequence of Theorem 9 from [8].

Proposition 7. Assume (P1). If there is $W \in l_{L(H, U)}^{\mathscr{E}}$ such that $T_{W}\left(t, t_{0}\right)$ is exponentially stable, then MARE (10) has a maximal solution $X_{\max }$ which has the property that

$$
R+\Pi_{3}\left(X_{\max }\right)>>0 .
$$

Theorems 9 and 13 from [8] ensure that, in the time invariant case, the notion of stabilizing solution of MARE (10) from [8] is equivalent with the one introduced by the following.

Definition 8. By a stabilizing solution of MARE (10), we mean a solution $\mathbb{X} \in l_{S(H)}^{\mathscr{I}}$ with the property that the evolution operator $T_{F^{\rtimes}}\left(t, t_{0}\right), t \geq t_{0}, t, t_{0} \in \mathbb{N}$, where

$$
F^{\mathbb{X}}=-\left(R+\Pi_{3}(\mathbb{X})\right)^{[-1]}\left(L+\Pi_{2}(\mathbb{X})\right)^{[*]}
$$

is exponentially stable.

Finally, we recall (see (4.8) in [8]) that mapping $\mathscr{R}$ can be equivalently rewritten as

$$
\begin{aligned}
\mathscr{R}(X)= & \Pi_{W}(X)+Q_{W} \\
& -\left(F^{X}-W\right)^{[*]}\left(R+\Pi_{3}(X)\right)^{[-1]}\left(F^{X}-W\right)
\end{aligned}
$$

for all $W \in l_{L(H, U)}^{\mathscr{Z}}$. We will use later this formula for different values of $W$.

\section{Main Results}

In this section we establish necessary (see Theorem 10) and sufficient conditions (see Theorems 11 and 13) for the existence of a stabilizing solution to MARE (10). They are related to the nonexistence of certain unobservable eigenvalues for a pair of operators which are defined with the coefficients of (10). For finite-dimensional MAREs, we recover the results from [17] (see also [11] for the continuous time case). Unlike [17], our results apply to stochastic systems with Markovian jumps.

Assume (P1) and let $W \in l_{L(H, U)}^{\mathscr{I}}$ and $\Pi_{W}, Q_{W}$ be defined by (28) and (29), respectively. Since $\Pi_{W}$ is a positive operator on $l_{S(H)}^{\mathscr{Z}}$, we know from [18] (see Theorem 3.2.3 therein) that there is $y^{*} \in\left(l_{S(H)}^{\mathscr{I}}\right)^{*}$ such that $y^{*} \geq 0, y^{*} \neq 0$, and

$$
\Pi_{W}^{*}\left(y^{*}\right)=\rho_{\Pi_{W}} y^{*}
$$

where $\rho_{\Pi_{W}}$ is the spectral radius of $\Pi_{W}$. Therefore, $\Pi_{W}^{*}$ has nonnegative eigenvalues.

Definition 9. We say that $\lambda \geq 0$ is an unobservable eigenvalue for the pair $\left(\Pi_{W}^{*}, Q_{W}\right), W \in l_{L(H, U)}^{\mathscr{Z}}$, iff there is an $y^{*} \in\left(l_{S(H)}^{\mathscr{Z}}\right)^{*}$ such that $y^{*} \geq 0, y^{*} \neq 0$, and

$$
\begin{aligned}
& \Pi_{W}^{*}\left(y^{*}\right)=\lambda y^{*}, \\
& y^{*}\left(Q_{W}\right)=0 .
\end{aligned}
$$

Otherwise, we will say that $\lambda$ is not an unobservable eigenvalue for the pair $\left(\Pi_{W}^{*}, Q_{W}\right)$.

The next theorem gives necessary conditions for the existence of a stabilizing solution to MARE (10). 
Theorem 10. If the algebraic Riccati equation (10) has a stabilizing solution, then

(a) $\Pi$ is stabilizable;

(b) $\lambda=1$ is not an unobservable eigenvalue for the pair $\left(\Pi_{-R^{-1} L^{[*]}}^{*}, Q_{-R^{-1} L^{[*]}}\right)$.

Proof. Statement (a) follows from Definition 8. Let us prove (b). If (10) has a stabilizing solution $\mathbb{X}$, then it coincides with the maximal solution, according to Theorem 13 from [8]. Since $0 \in \Gamma^{\Sigma}$, we deduce that $\mathbb{X} \geq 0$ (see Definition $5(\mathrm{~b})$ ). Using Theorem 13 from [8] again, we see that $\widetilde{\Gamma}^{\Sigma} \neq \varnothing$. A slight modification of the proof of Theorem 13 from [8] leads us to the conclusion that, in the time invariant case, $\widetilde{\Gamma}^{\Sigma}$ contains a constant sequence $X(t)=X \in l_{S(H)}^{\mathscr{I}}, t \in \mathbb{N}$. For such $X$ we have $\mathscr{R}(X)-X>>0$ and, consequently, there is a $\delta>0$ such that

$$
\mathscr{R}(X)-X \geq \delta \Phi_{H}
$$

Assume by contradiction that (b) is not satisfied. This means that there is $y^{*} \geq 0, y^{*} \neq 0$, such that

$$
\begin{aligned}
& \Pi_{-R^{-1} L^{[*]}}^{*}\left(y^{*}\right)=y^{*}, \\
& y^{*}\left(Q_{-R^{-1} L^{[*]}}\right)=0 .
\end{aligned}
$$

Using successively (36), (33) (with $-R^{-1} L^{[*]}$ replacing $W$ ), and (37), we get

$$
\begin{aligned}
& 0 \leq y^{*}(\mathscr{R}(X)-X)=y^{*}\left(\Pi_{-R^{-1} L^{[*]}}(X)-X\right. \\
& +Q_{-R^{-1} L^{[*]}}-\left(F^{X}+R^{-1} L^{[*]}\right)^{[*]}\left(R+\Pi_{3}(X)\right) \\
& \left.\cdot\left(F^{X}+R^{-1} L^{[*]}\right)\right)=\left(\Pi_{-R^{-1} L^{[*]}}^{*}\left(y^{*}\right)-y^{*}\right) X \\
& +y^{*}\left(Q_{-R^{-1} L^{[*]}}\right)-y^{*}\left[\left(F^{X}+R^{-1} L^{[*]}\right)^{T}\right. \\
& \left.\cdot\left(R+\Pi_{3}(X)\right)\left(F^{X}+R^{-1} L^{[*]}\right)\right] \\
& =-y^{*}\left[\left(F^{X}+R^{-1} L^{[*]}\right)^{T}\left(R+\Pi_{3}(X)\right)\right. \\
& \left.\cdot\left(F^{X}+R^{-1} L^{[*]}\right)\right] \leq 0 \text {. }
\end{aligned}
$$

Hence $y^{*}(\mathscr{R}(X)-X)=0$. From (36) and the positiveness of $y^{*}$, we deduce that

$$
0=y^{*}(\mathscr{R}(X)-X) \geq y^{*}\left(\delta \Phi_{H}\right) \geq 0
$$

It follows that $y^{*}\left(\delta \Phi_{H}\right)=0 \Leftrightarrow y^{*}\left(\Phi_{H}\right)=0$. Since $\left\|y^{*}(X)\right\| \leq\|X\|\left\|y^{*}\left(\Phi_{H}\right)\right\|$ for all $X \in l_{S(H)}^{\mathscr{E}}$, it follows easily that $y^{*}=0$. We have obtained a contradiction. Therefore, (b) holds and $\lambda=1$ is not an unobservable eigenvalue for the pair $\left(\Pi_{-R^{-1} L^{[*]}}^{*}, Q_{-R^{-1} L^{[*]}}\right)$. The proof is complete.
Now let us introduce a new hypothesis (i) $A_{p}(i) \in L(H)$ and $B_{p}(i) \in L(U, H), p=0, \ldots, r$,
$i \in \mathscr{Z}$, are compact operators.

In the case when $\mathscr{Z}$ is finite, we have the following converse of Theorem 10.

Theorem 11. Assume that $\mathscr{Z}$ is finite and (P1) and (P2) hold. If

(a) $\Pi$ is stabilizable and

(b) the pair $\left(\Pi_{-R^{-1} L^{[*]}}^{*}, Q_{-R^{-1} L^{[*]}}\right)$ has not an unobservable eigenvalue $\hat{\lambda}^{-1} \geq 1$, then the algebraic Riccati equation (10) has a stabilizing solution.

Proof. From Proposition 7, we know that the algebraic Riccati equation (10) has a maximal solution $X_{\max }$. Assume by contradiction that $X_{\max }$ is not stabilizing; that is, $T_{F^{x_{\max }}}\left(t, t_{0}\right)$ is not exponentially stable. Then, $\rho_{\Pi_{F} x_{\max }} \geq 1$ and there is $y^{*} \epsilon$ $\left(l_{S(H)}^{\mathscr{Z}}\right)^{*}, y^{*} \geq 0, y^{*} \neq 0$, such that $\Pi_{F^{X_{\max }}}^{*}\left(y^{*}\right)=\rho_{\Pi_{F} X_{\max }} y^{*}$. Hence, $y^{*}\left(\Pi_{F^{X_{\max }}}(X)\right)=\rho_{\Pi_{F} X_{\max }} y^{*}(X)$ for all $X \in l_{S(H)}^{\mathscr{F}^{x_{\max }}}$. For the sake of simplicity, let us denote $\rho_{\Pi_{F} X_{\max }}$ by $\bar{\rho}$.

Let us prove that $y^{*}\left(\mathcal{N}_{H}\right) \neq\{0\}$. Indeed, if $y^{*}\left(\mathcal{N}_{H}\right)=$ $\{0\}$, then $y^{*}\left(\overline{\mathscr{N}}_{H}\right)=0$. Since $\overline{\mathcal{N}}_{H}=\mathscr{C}_{H}^{\mathscr{I}}$, according to Lemma 1, we deduce that $y^{*}\left(\mathscr{C}_{H}^{\mathscr{I}}\right)=\{0\}$. On the other hand, hypothesis (P2) implies that $\Pi_{F^{x_{\max }}}(X) \in \mathscr{C}_{H}^{\mathscr{Z}}$ for all $X \in l_{S(H)}^{\mathscr{Z}}$ and, therefore, $\bar{\rho} y^{*}(X)=y^{*}\left(\Pi_{F^{x_{\max }}}(X)\right)=0$ for all $X \in$ $l_{S(H)}^{\mathscr{Z}}$. Hence, $y^{*}=0$ that is a contradiction. It follows that $y^{*}\left(\mathcal{N}_{H}\right) \neq\{0\}$.

Thus, $\left.y^{*}\right|_{\mathcal{N}_{H}}$ is a nonzero linear functional. Obviously, $\left|y^{*}(X)\right| \leq\|X\|_{\mathscr{E}}\left\|y^{*}\right\| \leq\||X|\|_{1}\left\|y^{*}\right\|, X \in \mathcal{N}_{H}$, and $\left.y^{*}\right|_{\mathcal{N}_{H}} \in$ $\left(\mathcal{N}_{H}\right)^{*}$. Moreover, $\left.y^{*}\right|_{\mathcal{N}_{H}} \geq 0,\left.y^{*}\right|_{\mathcal{N}_{H}} \neq 0$. By Proposition 3, we know that there is $Y \in l_{S(H)}^{\mathscr{E}}, Y \geq 0, Y \neq 0$, such that

$$
\left.y^{*}\right|_{\mathcal{N}_{H}}(X)=\sum_{i \in Z} \operatorname{Tr}[X(i) Y(i)]
$$

for all $X \in \mathcal{N}_{H}$. Rewriting (33) for $W$ replaced by $F^{X_{\max }}$, we see that the maximal solution $X_{\max }$ of the algebraic Riccati equation (10) satisfies the following equation:

$$
X_{\max }=\Pi_{F^{X_{\max }}}\left(X_{\max }\right)+Q_{F^{X_{\max }}} .
$$

Hence, $Q_{F^{X_{\max }}}=-\left(\Pi_{F^{X_{\max }}}\left(X_{\max }\right)-X_{\max }\right)$. From (P1)(i) and (29), $Q_{F^{X_{\max }}} \geq 0$. Using the properties of $y^{*}$, we obtain

$$
\begin{aligned}
0 & \leq y^{*}\left(Q_{F^{x_{\max }}}\right)=-y^{*}\left(\Pi_{F_{\max }}\left(X_{\max }\right)-X_{\max }\right) \\
& =-\left[\Pi_{F^{x_{\max }}}^{*}\left(y^{*}\right)-y^{*}\right]\left(X_{\max }\right) \\
& =-(\bar{\rho}-1) y^{*}\left(X_{\max }\right) \leq 0 .
\end{aligned}
$$


It follows that $y^{*}\left(Q_{F^{x_{\max }}}\right)=0$. An easy computation shows that

$$
\begin{aligned}
Q_{F^{X_{\max }}} & \\
= & M-L R^{[-1]} L^{[*]} \\
& +\left[R^{[-1]} L^{[*]}+F^{X_{\max }}\right]^{[*]} R\left[R^{[-1]} L^{[*]}+F^{X_{\max }}\right] .
\end{aligned}
$$

Lemma 28 from [8] (a Schur complement result for sequences of operators) and (P1)(i) imply that $M-L R^{[-1]} L^{[*]} \geq 0$. Since

$$
\left[R^{[-1]} L^{[*]}+F^{X_{\max }}\right]^{[*]} R\left[R^{[-1]} L^{[*]}+F^{X_{\max }}\right] \succeq 0
$$

and $Q_{-R^{-1} L^{[*]}}=M-L R^{[-1]} L^{[*]}$, it follows easily that

$$
y^{*}\left(Q_{-R^{-1} L^{[*]}}\right)=0
$$

and $y^{*}\left(\left[R^{[-1]} L^{[*]}+F^{X_{\max }}\right]^{[*]} R\left[R^{[-1]} L^{[*]}+F^{X_{\max }}\right]\right)=0$.

We know that, for any $X \in S(H)$ and $\left\{e_{n}\right\}_{n \in \mathbb{N}}$ an orthonormal basis of $H$, the increasing sequence $X_{n}=\sum_{n=1}^{\infty}\left(X e_{n}\right) \otimes$ $\left(X e_{n}\right) \in S_{1}(H)$ is weakly convergent to $X$. It is not difficult to see that this property remains valid for each component $X(i)$ of any $X \in l_{S(H)}^{\mathscr{X}}$. So there is a nonnegative, increasing sequence $R_{n} \in \mathcal{N}_{H}, n \in \mathbb{N}$ which is componentwise weakly convergent to $R$. The positiveness of $y^{*}$ and the inequality $R \geq R_{n} \geq 0$ imply that $y^{*}\left(\left[R^{[-1]} L^{[*]}+F^{X_{\max }}\right]^{[*]} R_{n}\left[R^{[-1]} L^{[*]}+\right.\right.$ $\left.\left.F^{X_{\max }}\right]\right)=0$. Since

$$
\left[R^{[-1]} L^{[*]}+F^{X_{\max }}\right]^{[*]} R_{n}\left[R^{[-1]} L^{[*]}+F^{X_{\max }}\right] \in \mathcal{N}_{H},
$$

we can apply (40) and we get

$$
\begin{aligned}
& \sum_{i \in \mathscr{F}} \operatorname{Tr}\left[Y(i)\left(\left(R^{[-1]} L^{[*]}+F^{X_{\max }}\right)(i)\right)^{*} R_{n}(i)\right. \\
& \left.\cdot\left(R^{[-1]} L^{[*]}+F^{X_{\max }}\right)(i)\right]=0 \\
& \Longleftrightarrow \sum_{i \in \mathscr{F}} \operatorname{Tr}\left[\left(\sqrt{Y(i)}\left(R^{[-1]} L^{[*]}+F^{X_{\max }}\right)(i)\right)^{*}\right. \\
& \left.\cdot R_{n}(i)\left(R^{[-1]} L^{[*]}+F^{X_{\max }}\right)(i) \sqrt{Y(i)}\right]=0
\end{aligned}
$$

for all $i \in \mathscr{Z}$. Now let us recall the following property of nonnegative operators: $\operatorname{Tr}\left[A A^{*}\right]=0 \Rightarrow A=0$. From (47), we obtain

$$
\begin{aligned}
& \sqrt{Y(i)}\left(R^{[-1]} L^{[*]}+F^{X_{\max }}\right)(i)^{*} \sqrt{R_{n}(i)} \\
& \quad=0 \Longrightarrow \sqrt{Y(i)}\left(R^{[-1]} L^{[*]}+F^{X_{\max }}\right)(i)^{*} R_{n}(i)=0 .
\end{aligned}
$$

Passing to the limit as $n \rightarrow \infty$ in the above equality, we get

$$
\sqrt{Y(i)}\left(R^{[-1]} L^{[*]}+F^{X_{\max }}\right)(i)^{*} R(i)=0 .
$$

Taking into account that $R(i)$ is invertible for all $i \in \mathscr{E}$, we deduce that $\sqrt{Y(i)}\left(R^{[-1]} L^{[*]}+F^{X_{\max }}\right)(i)^{*}=0$, for all $i \in \mathscr{Z}$ or, equivalently,

$$
\sqrt{Y}\left[R^{[-1]} L^{[*]}+F^{X_{\max }}\right]^{[*]}=0 .
$$

Thus, $\sqrt{Y}\left(A+B F^{X_{\max }}\right)^{[*]}=\sqrt{Y}\left(A-B R^{[-1]} L^{[*]}\right)^{[*]}$ for all $Y \in$ $l_{S(H)}^{\mathscr{I}}$ and

$$
\operatorname{Tr}\left[Y \Pi_{F^{X_{\max }}}(\widetilde{X})\right]=\operatorname{Tr}\left[Y \Pi_{-R^{[-1]} L^{[*]}}(\widetilde{X})\right]
$$

for all $\widetilde{X} \in \mathcal{N}_{H}$. In view of (40), we get

$$
\left.y^{*}\right|_{\mathcal{N}_{H}}\left(\Pi_{F^{X_{\max }}}(\widetilde{X})\right)=\left.y^{*}\right|_{\mathcal{N}_{H}}\left(\Pi_{-R^{[-1]} L^{[*]}}(\widetilde{X})\right)
$$

for all $\widetilde{X} \in \mathcal{N}_{H}$. Let us prove that the above equality remains true if we replace $\widetilde{X}$ with an arbitrary $X \in l_{S(H)}^{\mathscr{E}}$. As mentioned above, there is a sequence $\widetilde{X}_{n} \in \mathcal{N}_{H}$ that converges componentwise and weakly to $X$. That is, $\widetilde{X}_{n}(i) \underset{n \rightarrow \infty}{\rightarrow} X(i)$, weakly, for all $i \in \mathscr{Z}$. From hypothesis (P2), it follows that $\left(A_{p}+\right.$ $\left.B_{p} F^{X_{\max }}\right)^{*}(i) \mathfrak{E}\left(\widetilde{X}_{n}\right)(i)\left(A_{p}+B_{p} F^{X_{\max }}\right)(i)$ is norm convergent to $\left(A_{p}+B_{p} F^{X_{\max }}\right)^{*}(i) \mathfrak{F}(X)(i)\left(A_{p}+B_{p} F^{X_{\text {max }}}\right)(i)$ for all $i \in \mathscr{Z}$ and $p=0, \ldots, r$. This is because the multiplication to the right and to the left with compact operators promotes the weak convergence of operators to the uniform convergence.

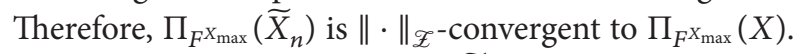
Similarly, we deduce that $\Pi_{\left.-R^{[-1]} L^{[*]}\right]}\left(\widetilde{X}_{n}\right)$ is $\|\cdot\|_{\mathscr{X}^{-} \text {-convergent }}$ to $\Pi_{-R^{[-1]} L^{[*]}}(X)$. From $(52)$ we have

$$
y^{*}\left(\Pi_{F^{X_{\max }}}\left(\widetilde{X}_{n}\right)\right)=y^{*}\left(\Pi_{-R^{[-1]} L^{[*]}}\left(\widetilde{X}_{n}\right)\right)
$$

and passing to the limit for $n \rightarrow \infty$ we obtain

$$
y^{*}\left(\Pi_{F^{X_{\max }}}(X)\right)=y^{*}\left(\Pi_{-R^{[-1]} L^{[*]}}(X)\right), \quad X \in l_{S(H)}^{\mathscr{I}} .
$$

So $\bar{\rho} y^{*}=\Pi_{F^{X_{\max }}}^{*}\left(y^{*}\right)=\Pi_{-R^{[-1]} L^{[*]}}^{*}\left(y^{*}\right)$. In view of (45), we just have obtained a contradiction of (b). Therefore, $X_{\max }$ is a stabilizing solution and the conclusion follows.

Remark 12. If the maximal solution would have the property that $\rho_{\Pi_{\mathrm{E}} X_{\max }} \leq 1$, the proof of the above theorem could be modified such that condition $\lambda \geq 1$ is to be replaced by $\lambda=1$.

Before stating the next result, we note that $A X, X A \in \mathcal{N}_{H}$ for all $A \in \mathcal{N}_{H}$ and $X \in l_{S(H)}^{\mathscr{E}}$; that is, $\mathcal{N}_{H}$ is a two-sided ideal of $l_{S(H)}^{\mathscr{I}}$.

In the case when $\mathscr{Z}$ is infinite, we have the following version of Theorem 11.

Theorem 13. Assume that $\mathscr{Z}$ is infinite and $A_{p}, B_{p} \in \mathcal{N}_{H}$, $p=0, \ldots, r$. If
(a) $\Pi$ is stabilizable and
(b) the pair $\left(\Pi_{-R^{-1} L^{[*]}}^{*}, Q_{\left.-R^{-1} L^{[*]}\right)}\right.$ has not an unobservable
eigenvalue $\lambda^{2} \geq$, then the algebraic Riccati equation (10) has a stabilizing solution.

Proof. Arguing as in the proof of the above theorem, we see that (10) has a maximal solution $X_{\max }$. Since

$$
\begin{gathered}
\Pi_{F^{X_{\max }}}(X)(i)=\sum_{p=0}^{r}\left(A_{p}(i)+B_{p} F^{X_{\max }}(i)\right)^{*} \mathfrak{E}(X)(i) \\
\cdot\left(A_{p}(i)+B_{p} F^{X_{\max }}(i)\right)
\end{gathered}
$$


for all $X \in l_{S(H)}^{\mathscr{I}}$ and $\mathcal{N}_{H}$ is a two-sided ideal of $l_{S(H)}^{\mathscr{E}}$, we deduce easily that

$$
\Pi_{F^{X_{\max }}}(X) \in \mathscr{N}_{H}
$$

for all $X \in l_{S(H)}^{\mathscr{E}}$. Assuming by contradiction that $X_{\max }$ is not a stabilizing solution and reasoning as in the proof of the above theorem, we deduce that $\rho_{\Pi_{F} X_{\max }} \geq 1$ and there is $y^{*} \epsilon$ $\left(l_{S(H)}^{\mathscr{I}}\right)^{*}, y^{*} \geq 0, y^{*} \neq 0$, such that $\Pi_{F^{x_{\max }}}^{*}\left(y^{*}\right)=\rho_{\Pi_{F} x_{\max }} y^{*}$. Property (56) ensures that $y^{*}\left(\mathcal{N}_{H}\right) \neq\{0\}$. Otherwise, (56) implies that $\rho_{\Pi_{F}^{*} X_{\max }} y^{*}(X)=y^{*}\left(\Pi_{F^{X_{\max }}}^{*}(X)\right)=0$ for all $X \epsilon$ $l_{S(H)}^{\mathscr{E}}$, which contradicts the assumption $y^{*} \neq 0$. As in the proof of Theorem 11, we obtain $y^{*}\left(Q_{-R^{-1} L^{[*]}}\right)=0$ and

$$
\begin{aligned}
& y^{*}\left(\left[R^{[-1]} L^{[*]}+F^{X_{\max }}\right]^{[*]} R\left[R^{[-1]} L^{[*]}+F^{X_{\max }}\right]\right) \\
& \quad=0 .
\end{aligned}
$$

Letting $Y=\psi^{-1}\left(\left.y^{*}\right|_{\mathcal{N}_{H}}\right)$ and repeating step by step the arguments in the proof of Theorem 11, we obtain successively $\sqrt{Y(i)}\left(R^{[-1]} L^{[*]}+F^{X_{\max }}\right)(i)^{*}=0, i \in \mathscr{Z}$, and $\sqrt{Y}\left(A+B F^{X_{\max }}\right)^{[*]}=\sqrt{Y}\left(A-B R^{[-1]} L^{[*]}\right)^{[*]}$ for all $Y \in$ $l_{S(H)}^{\mathscr{I}}$. Now, property (56) implies that $\operatorname{Tr}\left[Y \Pi_{F^{X_{\max }}}(X)\right]=$ $\operatorname{Tr}\left[Y \Pi_{-R^{-1} L^{[*]}}(X)\right]$ for all $X \in l_{S(H)}^{\mathscr{E}}$ and (54) holds. Therefore, $\Pi_{F^{X_{\max }}}^{*}\left(y^{*}\right)=\Pi_{-R^{-1} L^{[*]}}^{*}\left(y^{*}\right)$ and $\Pi_{-R^{-1} L^{[*]}}^{*}\left(y^{*}\right)=$ $\rho_{T_{\left(A-B F X_{\max )^{*}}\right.}} y^{*}$. Since $y^{*}\left(Q_{\left.-R^{-1} L^{[*]}\right)}\right)=0$, we have obtained a contradiction of statement (b). Consequently, $X_{\max }$ is a stabilizing solution and the proof is complete.

Now, let us apply our results to finite-dimensional MAREs associated with stochastic systems of the forms (5) and (6). We will prove that conditions (a) and (b) from Theorem 13 are necessary and sufficient for the existence of a stabilizing solution to MARE (10).

Corollary 14. Assume that $\mathscr{Z}$ is finite and the Hilbert spaces $H$ and $U$ are finite-dimensional. The algebraic Riccati equation (10) has a stabilizing solution if and only if

(a) $\Pi$ is stabilizable,

(b) $\lambda=1$ is not an unobservable eigenvalue for the pair $\left(\Pi_{-R^{-1} L^{[*]}}^{*}, Q_{-R^{-1} L^{[*]}}\right)$.

Proof. We first recall that, in finite dimensions, all linear and bounded operators are compact and nuclear. Also, if $\Pi$ is stabilizable, the maximal solution of MARE (10) has the property $\rho_{\Pi_{F} X_{\max }} \leq 1$. Indeed, from the proof of Theorem 9 from [8], we know that $X_{\max }(i)$ is the strong limit of an increasing sequence $X_{n}(i), i \in \mathscr{Z}$, of solutions of certain associated Lyapunov equations. Denoting by $\Pi_{1}^{n}$ the Lyapunov operators associated with these equations, we also know that $\Pi_{1}^{n}$ converges to $\Pi_{F^{x_{\max }}}$ and $\rho_{\Pi_{1}^{n}}<1$ (see $[7,8]$, e.g.,). Since the eigenvalues of a matrix and consequently its spectral radius depend continuously on the matrix coefficients, we pass to the limit as $n \rightarrow \infty$ in the last inequality and we get $\rho_{\Pi_{F} x_{\max }} \leq 1$. The conclusion follows.
Further, we observe that the hypotheses of Theorems 10 and 11 are fulfilled. The necessity part of the corollary follows from Theorem 10 while the sufficiency part is a direct consequence of Theorem 11 and Remark 12.

Remark 15. (a) Under the hypotheses of the above corollary, the operator $\Pi_{-R^{-1} L^{[*]}}^{*}$ coincides with the Lyapunov operator associated with the close-loop DTLS from [17, Theorem 12] for the stabilizing feedback controller $F=-R^{-1} L^{[*]}$.

(b) In view of (a), Corollary 14 shows that Theorems 10, 11 , and 13 are infinite-dimensional extensions of Theorem 12 from [17].

\section{Numerical Examples}

In this section, we provide some numerical examples which show the efficiency of our theory. Even in the finitedimensional case our results (see Corollary 14) seem to be new when applied to DTLSs with Markovian jumps. So, let us begin with a finite-dimensional example which proves that Corollary 14 is a viable alternative when stochastic detectability and stochastic observability conditions fail to hold.

Example 1. Consider (5) and (7) in the special case where the multiplicative noise is missing $\left(r=1, A_{1}=0, B_{1}=0\right)$ and

(P3) $H=U=\mathbb{R}, N \in \mathbb{N}^{*}, N>3$ is fixed, $\mathscr{Z}=\{1, \ldots, N\}$, $l_{S(H)}^{\mathscr{E}}=\mathbb{R}^{N}, A_{0}(i)=B_{0}(i)=3((i-1) / i), i \in \mathscr{Z}, M=$ $C^{[*]} C, C=(0,1,0,1,0,1, \ldots) \in \mathbb{R}^{N}, R(i)=1, L(i)=0$ for all $i \in \mathscr{Z}$, and $p_{i, i}=p_{i, i+1}=1 / 2$ for $i=1, \ldots, N-1$, $p_{N, N}=1$, and $p_{i, j}=0$ otherwise.

Let

$$
y(t)=C\left(\eta_{t}\right) x(t)
$$

be the output of (5). Note that, in its operation, system (5) switches between the modes

$$
\begin{aligned}
x(t+1)=3\left(\frac{i-1}{i}\right) x(t)+3\left(\frac{i-1}{i}\right) u(t), & \\
& \text { if } \eta(t)=i \in \mathscr{Z},
\end{aligned}
$$

according to the law of the Markov chain $\eta(t)$. For example, if the initial state is $\eta\left(t_{0}\right)=i$, it obeys (59) till time $t_{1}$ when the Markov chain switches to $\eta\left(t_{1}\right)=j \in \mathscr{Z}$ from $\eta\left(t_{0}\right)=i$ and system (5) obeys (59) with $j$ replacing $i$.

Let us solve the optimization problem $(\mathcal{O})$ defined in Section 1.

In this special case, problem $(\mathcal{O})$ is exactly a linear quadratic control problem associated with (5), (58). The associated MARE (10) has the following particular form:

$$
\begin{array}{r}
X(i)=4.5\left(\frac{i-1}{i}\right)^{2}(X(i)+X(i+1))+M(i) \\
-\frac{20.25((i-1) / i)^{4}(X(i)+X(i+1))^{2}}{1+4.5((i-1) / i)^{2}(X(i)+X(i+1))}, \\
i \in \mathscr{Z}-\{N\},
\end{array}
$$




$$
\begin{aligned}
X(N)= & 9\left(\frac{N-1}{N}\right)^{2}(X(N))+M(N) \\
& -\frac{81((N-1) / N)^{4}(X(N))^{2}}{1+9((N-1) / N)^{2}(X(N))} .
\end{aligned}
$$

We know [7] that if MAREs (60) and (61) have a stabilizing solution $\mathbb{X}$, then the optimal control problem ( $(\mathcal{)})$ has a solution $u(t)=F^{\mathbb{X}}(\eta(t)) x(t)$, where $F^{\mathbb{X}}$ is defined by (32). So we only have to find a stabilizing solution of (60) and (61).

Let $(A)$ be the stochastic system (5) without control (i.e., $\left.B_{p}=0, p=0, \ldots, r\right)$ and let $(A, C)$ be the system defined by $(A)$ and the output (58). The existing literature results (see [7] and the references therein) show that (10) has a stabilizing solution if the stochastic system with control is stabilizable and if $(A, C)$ is either stochastically observable or stochastically detectable. We will establish that $(A, C)$ is neither stochastically uniformly observable nor stochastically detectable. (Note that stochastic observability does not imply stochastic detectability [7].) In this case, we will see that Corollary 14 ensures the existence of the stabilizing solution.

(I) System $(A, C)$ Is Not Stochastically Uniformly Observable. According to Theorem 8 from [10], $(A, C)$ is stochastically uniformly observable iff there are $n_{0} \in \mathbb{N}$ and $\delta>0$ such that

$$
\sum_{k=0}^{n_{0}} \Pi_{1}^{k}\left(C^{[*]} C\right)(i)>\delta, \quad i \in \mathscr{Z},
$$

where $\Pi_{1}$ is defined by (22) with the coefficients from (P3). A direct computation shows that

$$
\begin{aligned}
\Pi_{1}(X)(i)=4.5\left(\frac{i-1}{i}\right)^{2}(X(i)+X(i+1)), & \\
i \in \mathscr{Z}-\{N\}, & \\
\Pi_{1}(X)(N) & =9\left(\frac{N-1}{N}\right)^{2} X(N),
\end{aligned}
$$

for all $X \in \mathbb{R}^{N}$. Using (P3), we see that $\Pi_{1}^{k}\left(C^{[*]} C\right)(1)=0$ for all $k \in \mathbb{N}$ and (62) fails to hold for $i=1$. Consequently, $(A, C)$ is not stochastically uniformly observable.

(II) System $(A, C)$ Is Not Stochastically Detectable. Following [5] (see Definition 2 and Proposition 16 therein), the system $(A, C)$ is detectable in conditional mean iff there is $F \in \mathbb{R}^{N}$ such that the nonnegative operator $\Pi_{F}^{C}$ defined by

$$
\begin{array}{r}
\Pi_{F}^{C}(X)(i) \\
=\left(3 \frac{i-1}{i}+F(i) C(i)\right)^{2}\left(\frac{X(i)+X(i+1)}{2}\right), \\
i \in \mathscr{Z}-\{N\}, \\
\Pi_{F}^{C}(X)(N)=\left(3 \frac{N-1}{N}+F(N) C(N)\right)^{2} X(N), \\
X \in \mathbb{R}^{N}
\end{array}
$$

generates an exponentially stable evolution operator. We observe that the matrix $\left(a_{i, j}\right)$ associated with $\Pi_{F}^{C}$ is upper triangular with the main diagonals $a_{i i}=(1 / 2)(3((i-1) / i)+$ $F(i) C(i))^{2}, i \in \mathscr{Z}-\{N\}$, and $a_{N N}=(3((N-1) / N)+$ $F(N) C(N))^{2}$. Since $a_{33}=2$, we deduce that $\lambda=2$ is an eigenvalue of $\Pi_{F}^{C}$ and $\rho_{\Pi_{F}^{C}} \geq 1$. So $\Pi_{F}^{C}$ cannot generate an exponentially stable evolution operator and $(A, C)$ is not detectable in conditional mean.

Now let us show that Corollary 14 can be used to prove that MARE (10) has a stabilizing solution in the special case when (P3) holds.

(III) Stabilizing Solution for the Algebraic Riccati Equation. We will prove that conditions (a) and (b) of Corollary 14 hold and, consequently, (60) has a stabilizing solution $\mathbb{X}$. Setting $W(i)=$ $-5 / 2, i \in \mathscr{Z}$, we have (see (28))

$$
\begin{aligned}
& \Pi_{W}(X)(i)=\frac{1}{4}\left(\frac{i-1}{i}\right)^{2}\left(\frac{X(i)+X(i+1)}{2}\right), \\
& i \in \mathscr{Z}-\{N\},
\end{aligned}
$$

$$
\Pi_{W}(X)(N)=\frac{1}{4}\left(\frac{N-1}{N}\right)^{2} X(N)
$$

for all $X \in \mathbb{R}^{N}$. The matrix associated with $\Pi_{W}$ is upper triangular. Since all the elements of the main diagonal are less than $1 / 4$, it follows that $\rho_{\Pi_{W}}<1 / 4$ and $\Pi_{W}$ is exponentially stable. Hence, $\Pi$ is stabilizable, according to Definition 8 , and condition (a) of Corollary 14 is satisfied. Let us prove that (b) holds, too. From (P3), we see that $Q_{-R^{-1} L^{[*]}}=M-L R^{[-1]} L^{[*]}=$

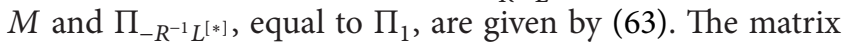
$\left(b_{i, j}\right)$ associated with $\Pi_{1}$ is also upper triangular with the main diagonal $b_{i, i}=4.5((i-1) / i)^{2}$ for $i \in \mathscr{Z}-\{N\}$ and $b_{N, N}=9((N-$ 1) $/ N)^{2}$. It is not difficult to see that $\lambda=1$ is not an eigenvalue of $\Pi_{1}$ because $1 \notin\left\{b_{i, i}, i \in \mathscr{Z}\right\}$. Consequently, $\lambda=1$ is not an eigenvalue of $\Pi_{1}^{*}=\Pi_{-R^{-1} L^{[*]}}^{*}$ and it cannot be an unobservable eigenvalue for the pair $\left(\Pi_{-R^{-1} L^{[*]}}^{*}, Q_{-R^{-1} L^{[*]}}\right)$. Condition (b) of Corollary 14 is satisfied and (60) has a stabilizing solution $\mathbb{X}$.

Proposition 14 from [10] ensures that this stabilizing solution is nonnegative and maximal among all nonnegative solutions of (60). Then, solving (60) and choosing the maximal solution, we get the stabilizing solution $\mathbb{X}$.

For $N=4$, the stabilizing solution is

$$
\begin{aligned}
& \mathbb{X}(1)=0, \\
& \mathbb{X}(2)=1.7446, \\
& \mathbb{X}(3)=0.84627, \\
& \mathbb{X}(4)=1.9061
\end{aligned}
$$

and the feedback stabilizing gain is

$$
\begin{aligned}
& F^{\mathbb{X}}(1)=0, \\
& F^{\mathbb{X}}(2)=-0.74455, \\
& F^{\mathbb{X}}(3)=0.84627, \\
& F^{\mathbb{X}}(4)=0.9061 .
\end{aligned}
$$


Then, the optimal control problem (G) has a solution $u(t)=$ $F^{\mathbb{X}}(\eta(t)) x(t)$, with the optimal cost being $J\left(u ; t_{0}, x, i\right)=$ $\langle\mathbb{X}(i) x, x\rangle, i \in\{1,2,3,4\}$.

In the following example, we apply Theorem 13 to prove the existence of stabilizing solutions for infinite-dimensional MAREs.

Example 2. Consider MARE (10) under the following additional hypotheses. The Hilbert space $H$ is infinitedimensional, $U=H, \mathscr{Z}=\mathbb{N}^{*}, r=1, A_{1}=B_{1}=0$, and $A_{0}(i)=B_{0}(i)=8\left((i-1) / i^{3}\right) K(i), i \in \mathbb{N}^{*}$, where $K \in l_{S_{1}(H)}^{\mathscr{}}$, $K \neq 0$. The other coefficients of MARE (10) are $L=0, R=$ $\Phi_{H}, M(1)=0, M(i)=I_{H}, i \in \mathscr{Z}, p_{i, i}=p_{i, i+1}=1 / 2, i \in \mathscr{Z}$, and $p_{i, j}=0$ otherwise. Let us show that the corresponding MARE (10) has a stabilizing solution.

Obviously $A_{0}, B_{0} \in \mathcal{N}_{H}, Q_{-R^{-1} L^{[*]}}=M$, and $\Pi_{-R^{-1} L^{[*]}}=$ $\Pi_{1}$. Assume that $\lambda \geq 1$ is an unobservable eigenvalue of $\left(\Pi_{-R^{-1} L^{[*]}}^{*}, Q_{-R^{-1} L^{[*]}}\right)$ and let $y^{*} \in\left(l_{S(H)}^{\mathscr{Z}}\right)^{*}$ be a positive eigenvector of $\lambda$. We have

$$
\begin{aligned}
\lambda\left(y^{*}\right)\left(I_{H}, 0,0, \ldots,\right) & =\Pi_{-R^{-1} L^{[*]}}^{*}\left(y^{*}\right)\left(I_{H}, 0,0, \ldots,\right) \\
& =y^{*}\left(\Pi_{1}\left(I_{H}, 0,0, \ldots,\right)\right) \\
& =y^{*}(0,0, \ldots,)=0 .
\end{aligned}
$$

It follows that $\left(y^{*}\right)(M)=y^{*}\left(\Phi_{H}\right)-\left(y^{*}\right)\left(I_{H}, 0,0, \ldots,\right)=$ $y^{*}\left(\Phi_{H}\right)$. From Lemma 6 from $[10]$, we know that $\left\|y^{*}\left(\Phi_{H}\right)\right\|=$ $\left\|y^{*}\right\| \neq 0$. Hence, $\left(y^{*}\right)\left(Q_{-R^{-1} L^{[*]}}\right)=\left(y^{*}\right)(M) \neq 0$ and we get a contradiction. Thus, $\lambda \geq 1$ cannot be an unobservable eigenvalue for the pair $\left(\Pi_{-R^{-1} L^{[*]}}^{*}, Q_{-R^{-1} L^{[*]}}\right)$. Condition (a) of Theorem 13 holds.

Further, we define $W(i)=-(1 /(2 k))(2 k+\sqrt{2}) I_{H}$, $i \in \mathscr{Z}$, where $k=\sup _{i \in \mathscr{Z}}\|K(i)\|_{1}$. Then, $\Pi_{W}\left(\Phi_{H}\right)(i)=$ $\left(1 /\left(2 k^{2}\right)\right)\left(8\left((i-1) / i^{3}\right)\right)^{2} K(i)^{*} K(i), i \in \mathbb{N}^{*}$, and $\left\|\Pi_{W}\left(\Phi_{H}\right)\right\|_{\mathscr{Z}} \leq$ $k^{2} \sup _{i \in \mathbb{N}^{*}}\left(1 / 2 k^{2}\right)\left(8\left((i-1) / i^{3}\right)\right)^{2}=1 / 2$. Therefore, $\left\|\Pi_{W}\right\|=$ $\left\|\Pi_{W}\left(\Phi_{H}\right)\right\|_{\mathscr{Z}} \leq 1 / 2$ and $\Pi_{W}$ generates an exponentially stable evolution operator. Thus, $\Pi$ is stabilizable, according to Definition 8. Conditions (a) and (b) of Theorem 13 are satisfied and (10) has a stabilizing solution. Theorem 9 from [8] provides an iterative algorithm for the computation of this stabilizing solution.

The following example is an application of Theorem 10.

Example 3. Assume that, except $A_{0}, B_{0}$, and $M$, the other coefficients of MARE (10) satisfy (P3) (see Example 1) with $N$ replaced by $\infty$. Let $A_{0}(i)=B_{0}(i)=1, M(i)=0$, for all $i \in \mathbb{N}^{*}$, and $\phi=(1, \ldots, 1, \ldots) \in l_{\infty}\left(\mathbb{N}^{*}\right)$. We recall that $\Pi_{-R^{-1} L^{[*]}}=$ $\Pi_{1}$ and $Q_{-R^{-1} L^{[*]}}=M$ and $\Pi_{1}$ is a positive operator. Since $\Pi_{1}(\phi)=\phi$, we can apply Lemma 6 from [10] to deduce that $\left\|\Pi_{1}\right\|=\left\|\Pi_{1}(\phi)\right\|_{\mathscr{Z}}=\|\phi\|_{\mathscr{Z}}=1$. On the other hand, $\lambda=1$ is an eigenvalue of $\Pi_{1}$ and, consequently, $\rho_{\Pi_{1}}=1$. Theorem 3.2.3 from [18] ensures that there is $y^{*} \in\left(l_{S(H)}^{\mathscr{Z}}\right)^{*}, y^{*} \geq 0$, $y^{*} \neq 0$, such that $\Pi_{-R^{-1} L^{[*]}}^{*}\left(y^{*}\right)=y^{*}$. Obviously, $y^{*}(M)=0$ and now it is clear that $\lambda=1$ is an unobservable eigenvalue for

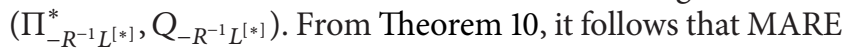
(10) has not a stabilizing solution.

\section{Conclusions and Further Research}

In this paper we have obtained necessary and sufficient conditions for the existence of stabilizing solutions for MARE (10) in infinite dimensions. These conditions are similar to those given in $[11,17]$ for finite-dimensional MAREs and do not involve other detectability and observability conditions. They are viable alternatives to existing results (see, e.g., [3, 5, 7] and the references therein) obtained under detectability or observability hypotheses.

The main difficulties in obtaining a "perfect" analog of the results from [17] (or [11]) are related to the fact that Lemma 1 is not true for infinite $\mathscr{Z}$ and we cannot ensure the existence of a nonnegative linear functional $y^{*}$ satisfying (34) and condition $y^{*}\left(\mathcal{N}_{H}\right) \neq\{0\}$. To compensate these gaps, we have assumed a compactness hypothesis in Theorem 11 and the condition $A_{p}, B_{p} \in \mathcal{N}_{H}, p=0, \ldots, r$, in Theorem 13. A natural question is how to relax these conditions.

Another open problem is whether the assertion $\rho_{\Pi_{F} X_{\max }} \leq$ 1 , where $X_{\max }$ is the maximal solution of MARE (10), remains true in infinite dimensions or for infinite $\mathscr{Z}$. The validity of this assertion will improve Theorems 11 and 13, as indicated in Remark 12.

Also, further research is required to study the existence of stabilizing solutions for MARE (10) in the case when the state space of the Markov chain is a general Borel Space as in [1].

\section{Conflict of Interests}

The author declares that there is no conflict of interests regarding the publication of this paper.

\section{References}

[1] O. L. V. Costa and D. Figueiredo, "LQ control of discrete-time jump systems with Markov chain in a general Borel space," IEEE Transactions on Automatic Control, 2014.

[2] M. D. Fragoso and J. Baczynski, "Optimal control for continuous-time linear quadratic problems with infinite Markov jump parameters," SIAM Journal on Control and Optimization, vol. 40, no. 1, pp. 270-297, 2001.

[3] O. L. V. Costa and M. D. Fragoso, "Discrete-time LQ-optimal control problems for infinite Markov jump parameter systems," IEEE Transactions on Automatic Control, vol. 40, no. 12, pp. 2076-2088, 1995.

[4] M. G. Todorov and M. D. Fragoso, "Output feedback $H_{1}$ control of continuous-time infinite Markovian jump linear systems via LMI methods," SIAM Journal on Control and Optimization, vol. 47, no. 2, pp. 950-974, 2008.

[5] V. M. Ungureanu, "Stability, stabilizability and detectability for Markov jump discrete-time linear systems with multiplicative noise in Hilbert spaces," Optimization, vol. 63, no. 11, pp. 16891712, 2014.

[6] O. L. V. Costa, M. D. Fragoso, and R. P. Marques, DiscreteTime Markov Jump Linear Systems, Springer Science \& Business Media, 2006.

[7] V. Dragan, T. Morozan, and A. Stoica, Mathematical Methods in Robust Control of Discrete Time Linear Stochastic Systems, Springer, New York, NY, USA, 2013. 
[8] V. M. Ungureanu, V. Dragan, and T. Morozan, "Global solutions of a class of discrete-time backward nonlinear equations on ordered Banach spaces with applications to Riccati equations of stochastic control," Optimal Control Applications and Methods, vol. 34, no. 2, pp. 164-190, 2013.

[9] V. M. Ungureanu and V. Dragan, "Stability of discrete-time positive evolution operators on ordered Banach spaces and applications," Journal of Difference Equations and Applications, vol. 19, no. 6, pp. 952-980, 2013.

[10] V. M. Ungureanu, "Optimal control for linear discrete-time systems with Markov perturbations in Hilbert spaces," IMA Journal of Mathematical Control and Information, vol. 26, no. 1, pp. 105-127, 2009.

[11] J. Zheng and L. Qiu, "On the existence of a mean-square stabilizing solution to a continuous-time Modi.ed algebraic Riccati equation," in Proceedings of the 21st International Symposium on Mathematical Theory of Networks and Systems, Groningen, The Netherlands, July 2014.

[12] D. L. Russell and G. Weiss, "A general necessary condition for exact observability," SIAM Journal on Control and Optimization, vol. 32, no. 1, pp. 1-23, 1994.

[13] B. Jacob and J. R. Partington, "The Weiss conjecture on admissibility of observation operators for contraction semigroups," Integral Equations and Operator Theory, vol. 40, no. 2, pp. 231243, 2001.

[14] H. H. Schaefer, Banach Lattices and Positive Operators, Springer, Berlin, Germany, 1974.

[15] C. S. Kubrusly, The Elements of Operator Theory, Birkhäuser, New York, NY, USA, 2nd edition, 2011.

[16] G. Da Prato and J. Zabczyk, Stochastic Equations in Infinite Dimensions, Cambridge University Press, Cambridge, UK, 1992.

[17] J. Zheng and L. Qiu, "On the existence of a mean-square stabilizing solution to a modified algebraic Riccati equation," in Proceedings of the 19th IFAC World Congress, vol. 19, part 1, pp. 6988-6993, International Federation of Automatic Control, 2014.

[18] T. Damm, Rational Matrix Equations in Stochastic Control, vol. 297, Springer, 2004. 


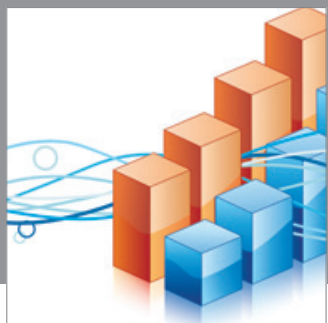

Advances in

Operations Research

mansans

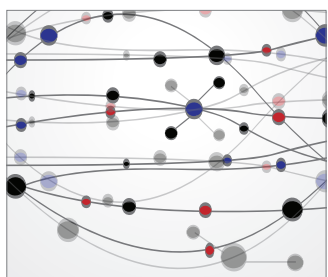

The Scientific World Journal
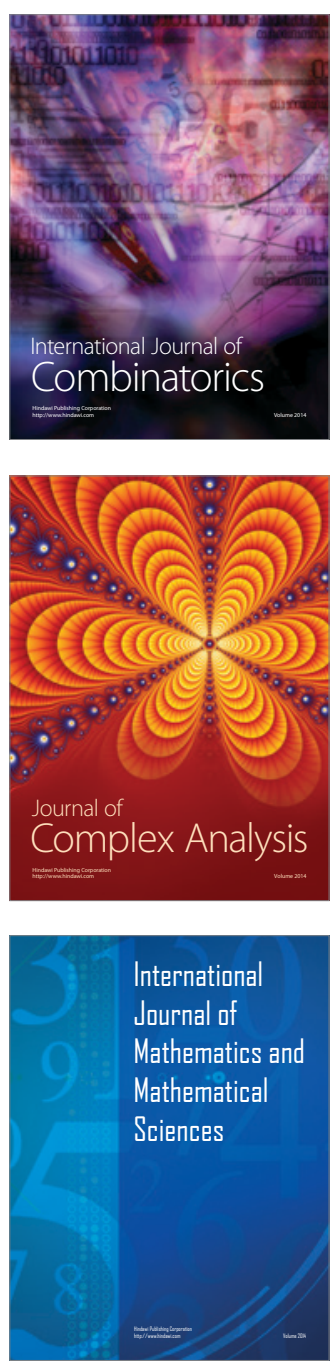
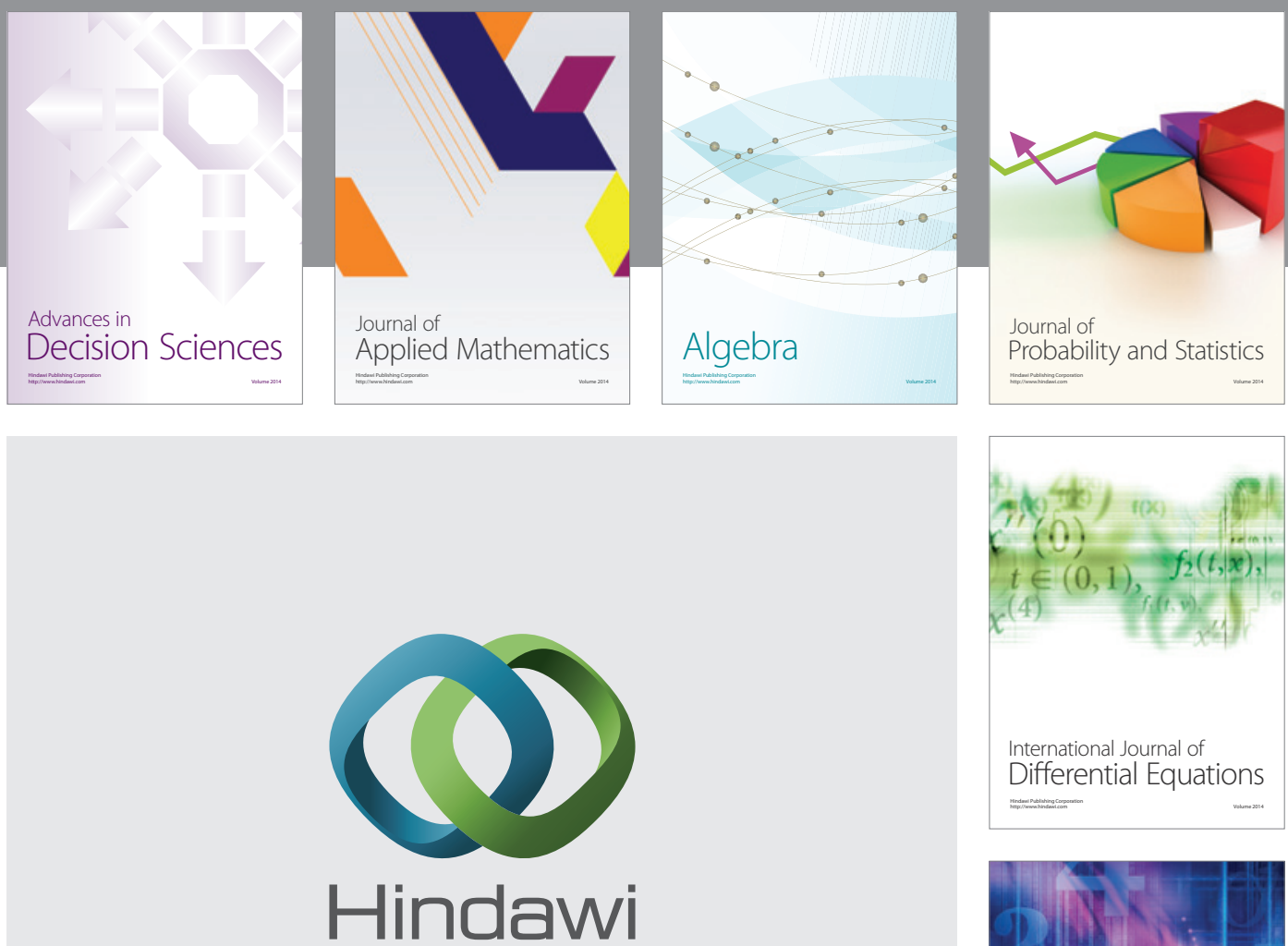

Submit your manuscripts at http://www.hindawi.com
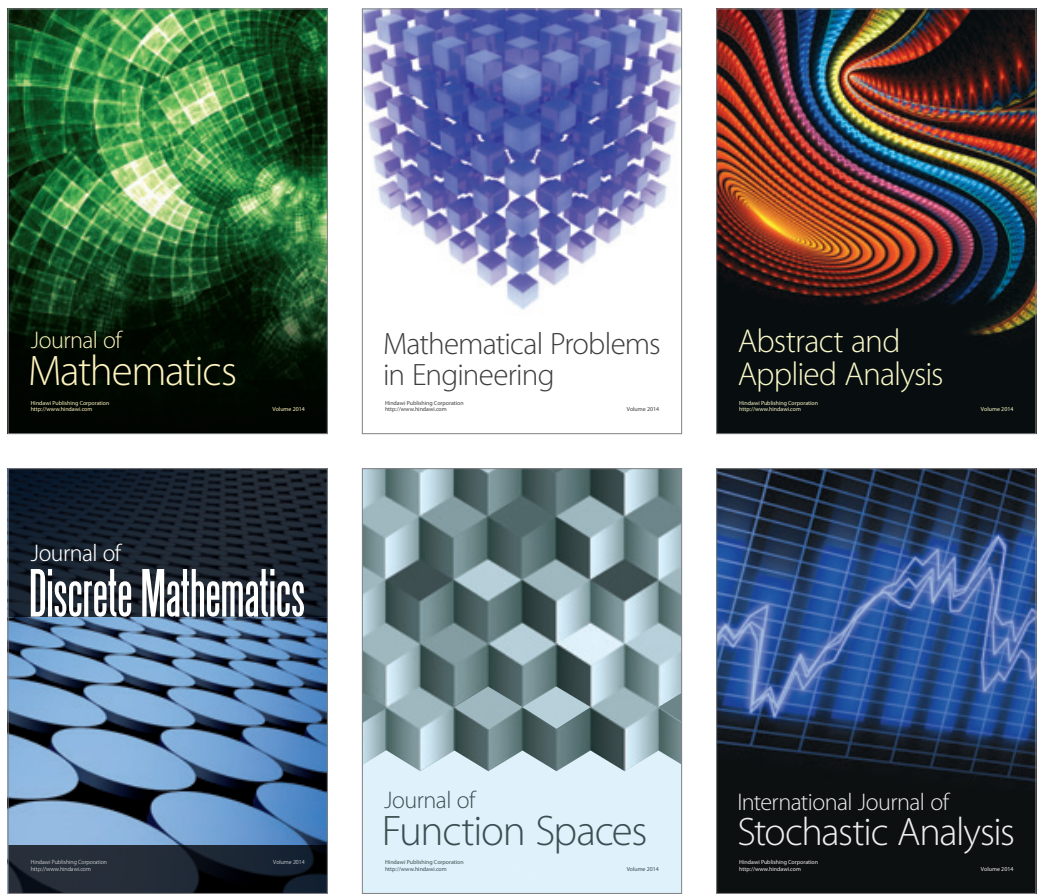

Journal of

Function Spaces

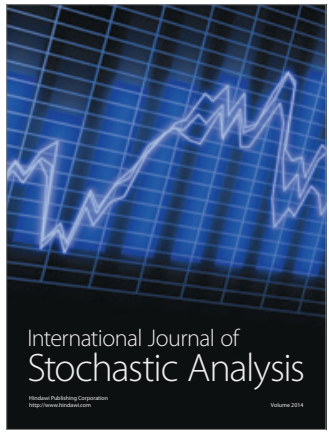

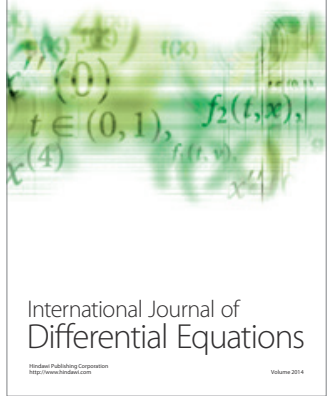
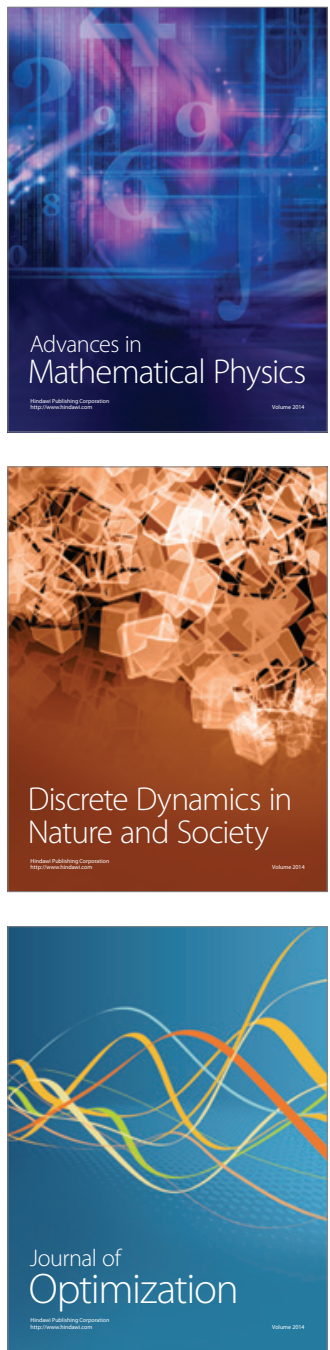